\title{
Systematics for types and effects of DNA variations
}

\author{
Mauno Vihinen(10
}

\begin{abstract}
Background: Numerous different types of variations can occur in DNA and have diverse effects and consequences. The Variation Ontology (VariO) was developed for systematic descriptions of variations and their effects at DNA, RNA and protein levels.

Results: VariO use and terms for DNA variations are described in depth. VariO provides systematic names for variation types and detailed descriptions for changes in DNA function, structure and properties. The principles of VariO are presented along with examples from published articles or databases, most often in relation to human diseases. VariO terms describe local DNA changes, chromosome number and structure variants, chromatin alterations, as well as genomic changes, whether of genetic or non-genetic origin.

Conclusions: DNA variation systematics facilitates unambiguous descriptions of variations and their effects and further reuse and integration of data from different sources by both human and computers.
\end{abstract}

Keywords: DNA variations, Mutation, Variation, Systematics, Variation ontology, VariO, Annotation, Databases, Ontology

\section{Background}

Variations at DNA are frequent and form the foundation of evolution. Some variants are related to diseases but many do not have any associated phenotype. The range of changes is very wide, from single nucleotide substitutions to changes in the number of entire chromosome sets. We can distinguish four categories, those in local DNA regions, such as genes; chromosomal variations; chromatin changes; and genome-wide alterations. To fully understand variants and their mechanisms and significance it is necessary to investigate them from different angles, e.g. to identify types of variants, but also to understand how they may affect structure, function, interactions, properties etc. For a systematic description of variations and their consequences, effects and mechanisms a framework called Variation Ontology (VariO) was developed [1]. As an ontology VariO facilitates systematic and detailed descriptions of variants. VariO includes terms for all kinds of alterations in DNA, RNA and protein.

Correspondence: mauno.vihinen@med.lu.se

Department of Experimental Medical Science, Lund University, BMC B13, SE-22184 Lund, Sweden

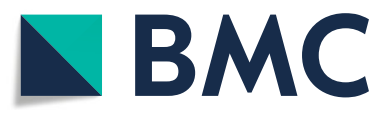

(c) The Author(s). 2018 Open Access This article is distributed under the terms of the Creative Commons Attribution 4.0 International License (http://creativecommons.org/licenses/by/4.0/), which permits unrestricted use, distribution, and

reproduction in any medium, provided you give appropriate credit to the original author(s) and the source, provide a link to the Creative Commons license, and indicate if changes were made. The Creative Commons Public Domain Dedication waiver (http://creativecommons.org/publicdomain/zero/1.0/) applies to the data made available in this article, unless otherwise stated.
Experimental studies provide the most reliable interpretation for variants and their effects and consequences. However, the huge volume of variants, e.g. about 3 million substitutions in a genome for a human individual, does not allow extensive experimental studies. Therefore, different kinds of prediction methods have been developed. The numbers of such tools are much higher for protein variants (see e.g. [2]). Non-coding variants are more difficult to predict largely due to lack of examples with known outcome. DeepSEA [3] is an example of a DNA predictor. For transcription factor binding sites and expression regulation, several approaches are available.

The Encyclopedia of DNA Elements (ENCODE) project has annotated functional elements at genomic regions, largely based on predictions [4]. There are data for transcription, transcription factor association, chromatin structure and histone modifications. For transcription factor binding sites and expression regulation, several predictors are available, reviewed in [5], that take into account sequence motifs, chromatin features and others. There are also methods to predict effects of cis regulatory elements and variants [6] including enhancers [7]. 
Dedicated methods are available for insertions and deletions whether affecting the reading frame or not [8-10]. When considering using these tools, one should bear in mind that most of them have not been systematically benchmarked as has been done for e.g. amino acid substitutions [11, 12]. Systematic method assessments are available for nucleosome position prediction methods $[13,14]$ as well as for predictors of topologically associating domains (TADs) [15].

Here, DNA variations, their types, functions, structural effects and properties are described in the systematic framework of VariO, similar to a previous article for protein variations [16]. As far as the author knows, this is the first systematic treatise of DNA variations and applicable to all organisms and kinds of variations and mechanisms. Variations at DNA level are important as such but also because they constitute the basis for inherited variations at RNA and protein levels. Examples are presented to highlight the different features of variants, usually in the context of human diseases.

\section{Databases for DNA variations}

Numerous databases distribute DNA variation information. In Table 1 [17-49] examples of some widely used resources and types of databases are given. All the human genes or numerous genes are represented in general variation databases while locus specific databases (LSDBs) are more focused and are collected for individual genes/diseases or groups of them. Many LSDBs are considered as the most reliable sources for disease related variation data, along with ClinVar. Exome and complete genome databases contain complete variation datasets from several studies. As these data are sensitive due to being personal, access is limited, however they are available for research purposes. Ethnic and national databases typically contain details for several diseases in more focused groups. For variation frequency information in diverse populations, dedicated resources are available and can be used e.g. for variation interpretation when finding out likely benign alterations.

Databases have been established for many diseases, those for cancer contain large amounts of data. Structural variants form a special group of alterations, there are specific data collections for them. Several resources share information on short repeat sequences and of methylation. Dedicated databases list microRNA and target variants, as well as DNA loops.

\section{Variation ontology}

For an efficient use, reuse, search and integration of variation information it is essential to describe it in a systematic way. VariO (http://variationontology.org/) was developed for the systematic description of variation types, effects, consequences and mechanisms [1]. The ontology is used to annotate information in databases at the three molecular levels: DNA, RNA and protein. Each of these levels contains further terms for variation type, function, structure and various properties. Here, DNA variation types and effects will be discussed. VariO annotations are always made in relation to a reference state, e.g. a reference sequence or a wild type property. A new version of VariO has been released with new terms, especially for DNA. VariO development continues, new terms are added and some rearrangements of already included terms are made when required, as in the latest releases for some areas in DNA and RNA terms. The basic structure of VariO has remained the same ever since first released, however new terms have been added, terms have been reorganized, clarified and redefined, when need has arisen. New terms, clarifications and updates can be suggested via the web site.

Systematic annotations consist of two parts: the VariO prefix and a number followed by the term. As an example, VariO:0132 is for "chromosomal variation". The number with the prefix is mandatory for annotation, the term name can be derived with that information. This article is organized according to the VariO: DNA variations are divided into the four major sublevels - DNA variation type, function, structure and properties. Subheadings are VariO terms, in the text terms are written in quotation marks. Detailed guidelines for the use and annotation have been published [50]. Consistent database annotations can be made with the VariOtator annotation tool [51]. VariO annotations are already used in a number of databases including some of those in the LOVD (Leiden Open (source) Variation Database) LSDB system, such as BTKbase [52] and SH2base [53], as well as in UniProt [54] and VariBench [55]. VariO is available in several ways including the website, AmiVariO, Ontology Lookup Service (https://www.ebi.ac.uk/ols/ontologies/vario), OBO Foundry (http://www.obofoundry.org/ontology/vario.html), NCBO BioPortal (https://bioportal.bioontology.org/ontol ogies/VARIO), Ontobee (http://www.ontobee.org/ontology/ VariO), AgroPortal (http://agroportal.lirmm.fr/ontologies/ VARIO), FAIRsharing (https://fairsharing.org/bsg-s000776/) and others.

VariO is used to describe the outcome of the mutation, i.e. the changed nucleotides etc., not the mechanism that led to the alteration. The latter we cannot explain just by looking at the variant. Note that "mutation" (VariO:0139) in VariO means "any process generating variation", not the outcome of these processes.

VariO annotations can be enriched with additional systematics, as described in the original article [1]. To 
Table 1 Examples of DNA variation databases

\begin{tabular}{|c|c|c|}
\hline Database & URL & Reference \\
\hline \multicolumn{3}{|l|}{ 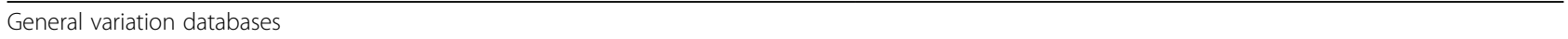 } \\
\hline Ensembl Variation Database & http://www.ensembl.org/info/genome/variation/index.html & {$[17]$} \\
\hline ClinVar & http://www.ncbi.nlm.nih.gov/clinvar/ & [18] \\
\hline Database of Short Genetic Variations (dbSNP) & http://www.ncbi.nlm.nih.gov/SNP/ & [19] \\
\hline \multicolumn{3}{|l|}{ Exome and complete genome sequences } \\
\hline EXAC & http://exac.broadinstitute.org & [20] \\
\hline NHLBI Exome Sequencing Project (ESP) Exome Variant Server (EVS) & http://evs.gs.washington.edu/EVS/ & [21] \\
\hline The 1000 Genomes Project & http://www.internationalgenome.org/ & [22] \\
\hline European Nucleotide Archive (ENA) & https://www.ebi.ac.uk/ena & [23] \\
\hline \multicolumn{3}{|l|}{ Locus specific variation databases } \\
\hline Leiden Open Variation Databases (LOVD) & http://www.lovd.nl/3.0/home & [24] \\
\hline Universal Mutation Database (UMD) & http://www.umd.be/ & [25] \\
\hline ImmunoDeficiency Variation Databases (IDbases) & http://structure.bmc.lu.se/idbase & [26] \\
\hline The TP53 web site & http://www.p53.fr/ & [48] \\
\hline \multicolumn{3}{|l|}{ Allele frequency databases } \\
\hline The ALlele FREquency Database (ALFRED) & https://alfred.med.yale.edu/alfred/ & [27] \\
\hline FINDbase & http://www.findbase.org/ & [28] \\
\hline Allele Frequency Net Database (AFND) & http://www.allelefrequencies.net/ & [29] \\
\hline Allele Frequency Community (AFC) & http://www.allelefrequencycommunity.org/ & [30] \\
\hline \multicolumn{3}{|l|}{ Cancer variation databases } \\
\hline Catalogue of Somatic Mutations in Cancer (COSMIC) & http://cancer.sanger.ac.uk/cosmic & [31] \\
\hline The Cancer Genome Atlas (TCGA) & https://portal.gdc.cancer.gov/ & [32] \\
\hline International Cancer Genome Consortium (ICGC) & https://dcc.icgc.org/ & [33] \\
\hline \multicolumn{3}{|l|}{ Ethnic/national databases } \\
\hline Pakistan Genetic Mutation Database & http://www.pakmutation.com/ & [34] \\
\hline The Singapore Human Mutation And Polymorphism Database & http://shmpd.bii.a-star.edu.sg/ & [35] \\
\hline \multicolumn{3}{|l|}{ Databases of genomic structural variations } \\
\hline dbVar & https://www.ncbi.nlm.nih.gov/dbvar/content/human_hub/ & {$[36]$} \\
\hline Database of Genomic Variants (DGV) & http://dgv.tcag.ca/dgv/ & [37] \\
\hline Database of Genomic Variants archive (DGVa) & https://www.ebi.ac.uk/dgva & [38] \\
\hline Mitelman Database of Chromosome Aberrations in Cancer & http://cgap.nci.nih.gov/Chromosomes/Mitelman & \\
\hline Human Polymorphic Inversion Database (InvFEST) & http://invfestdb.uab.cat/ & [39] \\
\hline \multicolumn{3}{|l|}{ Repeat databases } \\
\hline The European database of L1-HS retrotransposon insertions in humans (euL1db) & http://eul1db.unice.fr/ & [40] \\
\hline L1base, LINE-1 insertions & http://11base.charite.de/l1base.php & [41] \\
\hline SINEbase & http://sines.eimb.ru/ & [42] \\
\hline Short Tandem Repeat DNA Internet DataBase (STRBase) & https://strbase.nist.gov/ & [43] \\
\hline \multicolumn{3}{|l|}{ Methylation databases } \\
\hline Methylation Bank (MethBank) & http://bigd.big.ac.cn/methbank & [49] \\
\hline NGSmethDB & http://bioinfo2.ugr.es:8888/NGSmethDB/ & [44] \\
\hline \multicolumn{3}{|l|}{ miRNA target databases } \\
\hline Polymorphism in microRNAs and their TargetSites (PolymiRTS) & http://compbio.uthsc.edu/miRSNP/ & [45] \\
\hline Somatic mutations altering microRNA-ceRNA interactions (SomamiR DB) & http://compbio.uthsc.edu/SomamiR/ & [46] \\
\hline \multicolumn{3}{|l|}{ DNA loop database } \\
\hline R-loop DB & http://rloop.bii.a-star.edu.sg/ & [47] \\
\hline
\end{tabular}


provide details on the methods based on which the annotations are made, Evidence \& Conclusion Ontology (ECO) terms [56] can be used to indicate whether and which laboratory experiments, computational methods, literature curation, or other means have been applied.

\section{DNA variation type (VariO:0129)}

Variation type in VariO provides a description of a variation in English (see Fig. 1). Variation type terms provide a brief description with commonly used terms. They are not intended to replace Human Genome Variation Society (HGVS) names [57] or the International System for human Cytogenetic Nomenclature (ISCN) [58], instead to provide an easily understandable description for human readers and computer applications. VariO terms can be used together with HGVS and ISCN nomenclature.

There are four levels for the descriptions: DNA chain, chromosomal, genomic and chromatin levels, depending on the type and size of the variation. With VariOtator, the variation type annotations at DNA, RNA and protein level can be made automatically, including for Leiden Open Variation databases (LOVD), from the HGVS names. In the following examples, HUGO Gene Nomenclature Committee (HGNC) names [59] are indicated for genes. The HGVS prefixes for DNA (c. for coding DNA, g. genomic sequence, $\mathrm{m}$. mitochondrial) are used in the text. In some instances protein variants are discussed, they are indicated with prefix $\mathrm{p}$.

\section{DNA variation classification (VariO:0322)}

Histone variants or alterations in remodeler and modifier enzymes or their expression affect "chromatin variation" (VariO:0153). These alterations are frequent in cancers [60]. "Chromosomal variation" (VariO:0132) is either "variation of chromosome number" (VariO:0133) or "variation of chromosome structure" (VariO:0134). Down syndrome with trisomy of chromosome 21 [61] is an example of "variation of chromosome number", while Rett syndrome due to an inversion in X chromosome [62] is a "variation of chromosome structure".

Variations in the DNA chain occur e.g. within a gene or another functional unit, while chromosomal variations affect larger regions in chromosomes. The different types of DNA chain variations in a short sequence are shown in Fig. 2. Chromosomal variations are described in detail with "variation affecting DNA structure" (VariO:0155) annotations.

There are 5 categories of "DNA chain variation" (VariO:0135) types, some of them with subcategories. "DNA deletion" (VariO:0141) of G from region for intron 3 (g.101374535del) in BTK gene coding for Bruton tyrosine kinase causes a splice defect and leads to X-linked agammaglogulinemia (XLA) [63]. "DNA indel" (VariO:0143) is a variant that is due to both insertion and deletion. Alteration from $\mathrm{C}$ to TG in BTK gene coding for exon 17 (c.1684_1685delinsT) causes XLA due to RNA frameshift and truncated protein [64] is an example of a DNA indel. The original base $C$ is deleted and TG inserted instead. "DNA insertion" (VariO:0142) introduces a new base(s) to the DNA, such as insertion of T to BTK gene for exon 3 (g.101374623insT) introducing a new stop codon [65]. "DNA substitution" (VariO:0136) is the most common single nucleotide variation type and DNA variation in general. $\mathrm{G}$ to $\mathrm{C}$ substitution in the $B T K$ gene coding for the TH domain (g.101362620C > G) causes amino acid substitution in Zn finger leading to XLA [66]. DNA substitutions are either transitions or transversions. "Transition" (VariO:0313) changes a purine base (A, G) to another purine or a pyrimidine $(\mathrm{C}, \mathrm{T})$ to another pyrimidine. "Transversion" (VariO:0316) is a substitution from a purine to pyrimidine or vice versa. The $\mathrm{G}$ to $\mathrm{C}$ substitution is a transversion. Transitions can be classified further to "purine transition" (VariO:0315) and "pyrimidine transition" (VariO:0314) .

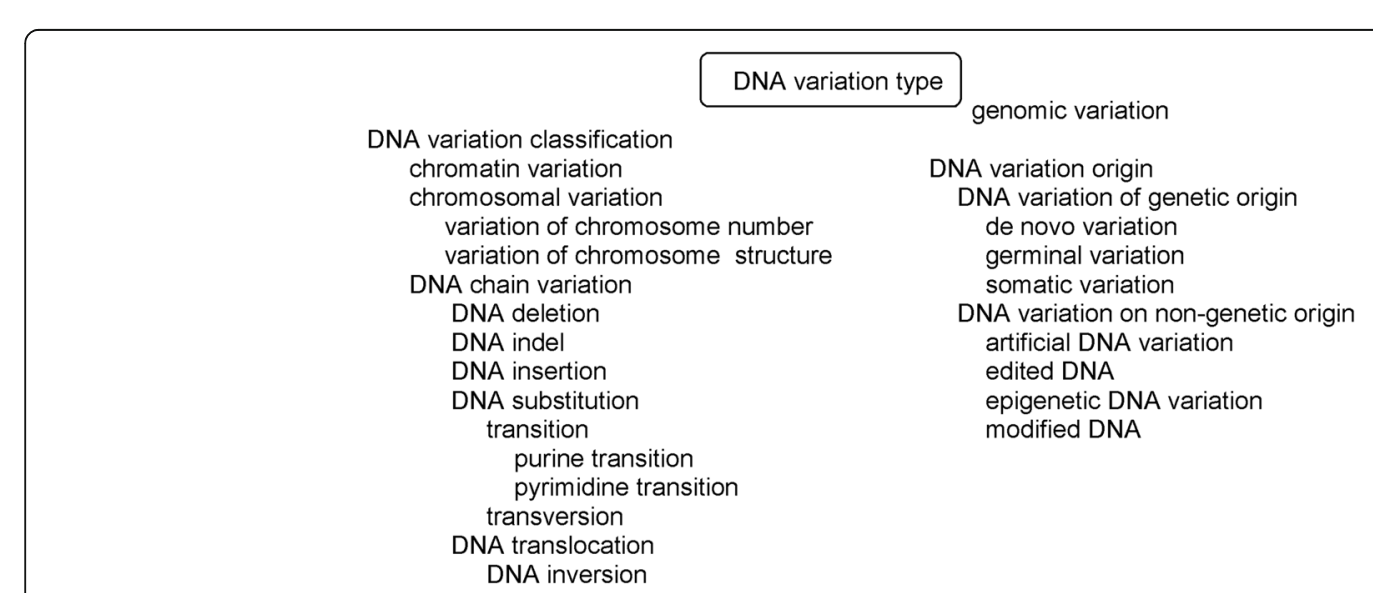

Fig. 1 Terms to describe DNA variation types 


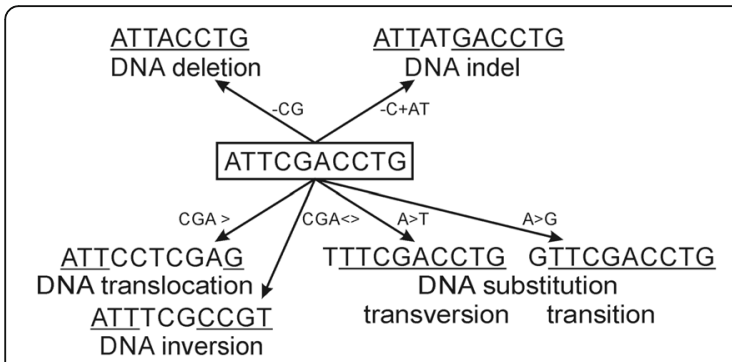

Fig. 2 Examples of DNA chain variations. The original sequence is in the middle. In the variant sequences the original bases at original positions are underlined

When a sequence stretch is moved to a new location within a chromosome it is called for "DNA translocation" (VariO:0144). "DNA inversion" (VariO:0145) is a special type of translocation where the sequence is inverted to its original place. Microinversions are rare, such as a 95 nucleotide inversion at 22q11.21 (Database of Genomic Variants nsv1129408) [67].

Genomic variations affect the entire genome. Autopolyploidy, which means duplication of chromosome sets originating from the same organism, is an example of "genomic variation" (VariO:0131) and common in human liver [68].

\section{DNA variation origin (Vario:0127)}

There are two types of "DNA variation origin" (VariO:0127), namely "DNA variation of genetic origin" (VariO:0130) and "DNA variation of non-genetic origin" (VariO:0146). Variants of genetic origin have appeared on DNA (or RNA) level and therefore directly affect the protein, when in a coding region.

Insertion in the non-coding region of exon 2 in $B T K$ is a "de novo variation" (VariO:0444) and has occurred in that invididual [69], while $G$ to $C$ substitution (c.1685G > C) for codon 562 causing p.R562P substitution in protein is a "germinal variation" (VariO:0445) [70] that has occurred in the germ cell of the mother. Melanoma-related A to $\mathrm{T}$ transversion in GNA11 (G protein subunit alpha 11) gene leading to a G209L substitution is a "somatic variation" (VariO:0446) [71].

Several variation types are of non-genetic origin. Replacement of A by C in BTK leading to the amino acid substitution p.Y334S was made in a construction and is thus an "artificial DNA variation" (VariO:0172) [72]. Novel genome editing technologies allow generation of specific DNA alterations e.g. to correct for genetic defects as in $\beta$-thalassemia [73] leading to "edited DNA" (VariO:0407). This example is an artificial variation, but genomic editing appears naturally in some organisms. Changes in DNA methylation pattern are a form of "epigenetic DNA variation" (VariO:0147) and are associated to systemic lupus erythematosus due to changes in transcription activation [74]. DNA lesion, such as incorporation of 8-hydroxyguanine to DNA, causes a form of "modified DNA" (VariO:0337) [75].

\section{Variation affecting DNA function (VariO:0148)}

DNA molecules have several functions. Some DNA molecules have catalytic deoxyribozyme activities. Self-catalyzed sequence-specific DNA depurination is the only known DNA catalytic activity [76]. Variations to the required cruciform structure could have an "effect on catalytic DNA activity" (VariO:0412).

Deletion of $\mathrm{G}$ from the region for intron 3 in BTK gene causes splice defect and XLA [63] due to "effect on DNA information transfer" (VariO:0150). The type of DNA variation affects DNA repair mechanisms. T/G or U/G mismatches are corrected by base excision repair, but lead also to increased frequency of variations i.e. have an "effect on DNA repair" (VariO:0151) as reviewed in [77]. Variation A to $\mathrm{C}$ in the TATA box of the $H B B$ gene for hemoglobin subunit beta leads to $\beta$-thalassemia [78] because of "effect on regulatory function of DNA" (VariO:0152). DNA replication fidelity can be affected by numerous factors including DNA variations such as DNA adducts caused by reactions with e.g. environmental mutagens, and sequence context $[79,80]$, thus having an "effect on DNA replication" (VariO:0154).

Variations at two major TERT (telomerase reverse transcriptase) gene promoter sites are frequent in melanoma patients and generate binding sites for Ets/TCF transcription factors [81]. These variants are classified to have "effect on transcription" (VariO:0149).

\section{Variation affecting DNA property (Vari0:0227)}

DNA properties affected by variations are described by terms in this category. Insertion of $\mathrm{T}$ to the $B T K$ gene coding for exon 3 introduces a new stop codon [65] and has "association of DNA variation to pathogenicity" (VariO:0229). Variation c.82C $>\mathrm{T}$ in BTK causing p.R28C [82] affects "conservation of DNA variation site" (VariO:0231) [83] by affecting highly conserved position. Variations at TERT gene promoter in melanoma patients generate binding sites for Ets/TCF transcription factors [81] and have "effect on DNA interaction" (VariO:0230).

\section{Variation affecting DNA structure (VariO:0155)}

DNA structure and architecture have several levels and layers. In addition to the double stranded form there are single and multiple stranded DNA forms and with and without RNA. Depending on the cell cycle stage, the DNA chain condensation varies greatly from a very tightly packed form to an elongated DNA chain. The entire structure of this most extended part of DNA terms is depicted in Fig. 3. 
variation affecting DNA structure

affected DNA level

chromosome affected

DNA chain affected

variation at intergenic DNA

variation in exon

variation in intron

extrachromosomal DNA affected

extrachromosomal circular DNA

organellar DNA affected

mitochondrial DNA affected

plastid DNA affected

plasmid affected

genome affected

chromatin structure variation

nucleosome positioning

variation at topology associating domain

chromosome variation

chromosome number variation

disomy

uniparental disomy

heterodisomy

isodisomy

monosomy

nullisomy

polysomy

tetrasomy

trisomy

chromosome structure variation

chromosomal amplification

copy number variation

DNA mobile genetic element insertion

DNA transposon

insertion sequence

retrotransposon insertion

LINE

LTR

SINE

nucleotide expansion

microsatellite

dinucleotide expansion

heptanucleotide expansion

mononucleotide expansion

pentanucleotide expansion

tetranucleotide expansion

trinucleotide expansion

minisatellite

type of chromosomal amplification

interspersed repeat

tandem repeat

chromosomal deletion

copy number variation

interstitial deletion

terminal deletion

chromosomal translocation

interchromosomal translocation

dicentric translocation

reciprocal chromosomal translocation

Robertsonian translocation

intrachromosomal translocation

chromosomal inversion

paracentric inversion pericentric inversion

complex chromosomal variation

immunological recognition molecule

diversification

class switch recombination

immunological receptor gene conversion

immunological receptor gene -

rearrangement

somatic hypermutation

isochromosome

ring chromosome

telomere length change

telomere extension

telomere shortening

DNA sugar variation

effect on DNA tertiary structure

effect on DNA form

effect on DNA double helix

effect on A DNA

effect on $B$ DNA

effect on C DNA

effect on D DNA

effect on L DNA

effect on S DNA

effect on Z DNA

effect on DNA triple helix

effect on intermolecular DNA triple helix

effect on D loop

effect on intramolecular DNA triple -

helix

effect on H DNA

effect of four-stranded DNA

effect on DNA cruciform

effect on i motif

effect on nucleic acid G-quadruplex

effect on single stranded DNA structure effect on A motif

effect on DNA-RNA hybrid

effect on $D$ loop

effect on $R$ loop

effect on T loop

epigenetic DNA modification

epigenetic DNA methylation

histone modification

nucleosome positioning

genome variation

chromosome set number variation

diploidy

hexaploidy

monoploidy

nulliploidy

pentaploidy

polyploidy

allopolyploidy

autopolyploidy

tetraploidy

triploidy

complex aenomic variation

Fig. 3 Terms for describing variations affecting DNA structure 


\section{Affected DNA level (VariO:0159)}

DNA level terms are used to indicate what kind of DNA molecule and region is affected by the variation. A Rett syndrome-causing inversion in the $\mathrm{X}$ chromosome [62] has "chromosome affected" (VariO:0164). "DNA chain affected" (VariO:0160) has three subcategories. TERT gene promoter variants in melanoma patients that generate binding sites for Ets/TCF transcription factors [81] are "variation at intergenic DNA" (VariO:0163). G to $C$ substitution in the BTK gene leads to amino acid substitution at zinc finger motif causing XLA [66] and is a "variation in exon" (VariO:0162). Deletion of G from the region for intron 3 in the BTK gene causes splice defect and XLA [63] and is a "variation in intron" (VariO:0161) .

G to A substitution coding for codon 467 (p.A467T) in the mitochondrial POLG (DNA polymerase gamma, catalytic subunit) gene causing progressive external opthalmoplegia and other diseases [84] has "extrachromosomal DNA affected" (VariO:0072) of type "organellar DNA affected" (VariO:0448) and even more specifically "mitochondrial DNA affected" (VariO:0450). Mitochondria are essential organelles for energy production in eukaryotes, whereas the other compartments with their own DNA, plastids are unique for plants and algae and appear only in some eukaryotes. Substitutions in the plastid infA (IF1 homolog) gene in spring barley lead to cytoplasmic line 2 (CL2) syndrome [85] and have "plastid DNA affected" (VariO:0451).

There are two additional forms of "extrachromosomal DNA affected" (VariO:0072). Variants to a H group plasmid change its maintenance as temperature sensitive in Escherichia coli [86]. In this case the variant has "plasmid affected" (VariO:0391). Plasmids are independently replicating circular DNA units common in bacteria but can appear also in other organisms. Plasmids can be transferred between cells, even organisms. Many plasmids contain toxin or antibiotic resistance genes. "Extrachromosomal circular DNA" (VariO:0449) is common in many organisms and are widely variable in size and contents as they originate from material in linear chromosomes [87].

Trisomy of chromosome 21 [61] has "genome affected" (VariO:0391).

\section{Chromatin structure variation (VariO:0226)}

GAA triplet expansions in the $F X N$ (frataxin) gene are the most usual cause of Friedreich ataxia, a form of progressive damage of the nervous system. The triplet expansion alters nucleosome positioning so that transcriptional activity is reduced because the start site is not accessible [88] being a "chromatin structure variation" (VariO:0226) due to effect on "nucleosome positioning" (VariO:0158).
Topologically associating domains (TADs) are a higher order chromatin structures where genomic regions interact with each other. These regions are thought to be involved e.g. in regulation. "Variation in topology associating domain" (VariO:0454) appears in diseases including various forms of cancers where boundaries of TADs are altered [89].

\section{Chromosome variation (Vario:0176)}

"Chromosome variation" (VariO:0176) is divided into two categories "chromosome number variation" (VariO:0206) and "chromosome structure variation" (VariO:0180).

\section{Chromosome number variation (VariO:0206)}

Variations in this category are used to describe changes in the number of chromosomes. "Nullisomy" (VariO:0212), lack of both chromosomal pairs, is not viable in human. "Disomy" (VariO:0208) is the normal genetic setting e.g. in human. Prader-Willi syndrome is caused by a lack of expression of genes in paternal chromosome in a segment of chromosome 15. There are three mechanisms behind the condition, one of them is "uniparental disomy" (VariO:0209) [90]. "Heterodisomy" (VariO:0211) appears when the non-identical chromosomes are from one parent. Paternal heterodisomy in chromosome 1 involving the LYST (lysosomal trafficking regulator) gene containing a substitution introducing a stop codon on RNA causes Chediak-Higashi syndrome [91]. In "isodisomy" (VariO:0210) there is a duplication of a single chromosome from one parent. Paternal genome-wide "uniparerental disomy" (VariO:0209), a condition where both copies of a chromosome or its part in a diploid cell or organism are from just one parent, in a patient causes Beckwith-Wiedemann syndrome [92]. Down syndrome is caused because of complete or partial "trisomy" (VariO:0207) of chromosome 21 [61] (Fig. 4a). In "tetrasomy (VariO:0213)" there are four copies of the chromosome and in "polysomy" (VariO:0303) more than the normal number.

\section{Chromosome structure variation (VariO:0180)}

The numerous types of variations in this category are depicted in Fig. 4.

Chromosomal amplification (VariO:0183) Numerous variation types and mechanisms affect the number of chromosomal region copies. "Copy number variation" (CNV) (VariO:0187) ranges in size from $1 \mathrm{~kb}$ up to several megabases and can be either amplification or deletion (Fig. 4b). CNV duplication of LAMB1 (laminin B1) gene causes autosomal dominant leukodystrophy [93].

DNA mobile genetic element insertion (VariO:0192) "DNA mobile genetic element insertion" (VariO:0192) 
A

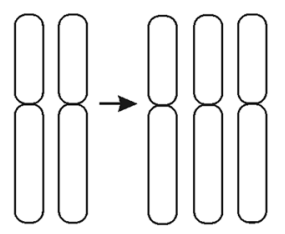

$\mathbf{F}$

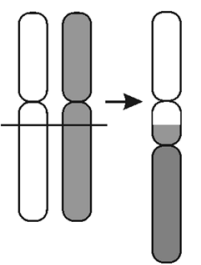

B

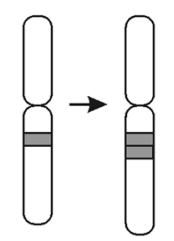

G

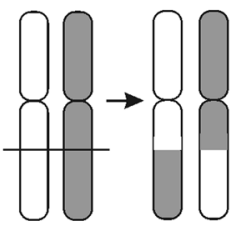

C

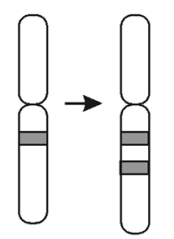

H

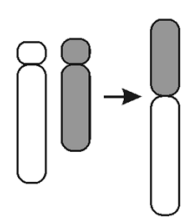

D<smiles>O=C(CC1CC1)N1CCCC1</smiles>

I

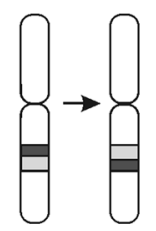

E<smiles>O=C1CCCCC1C1CC1</smiles>

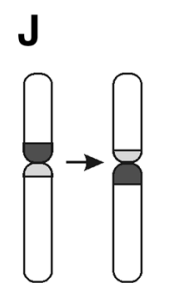

K

$\mathbf{L}$
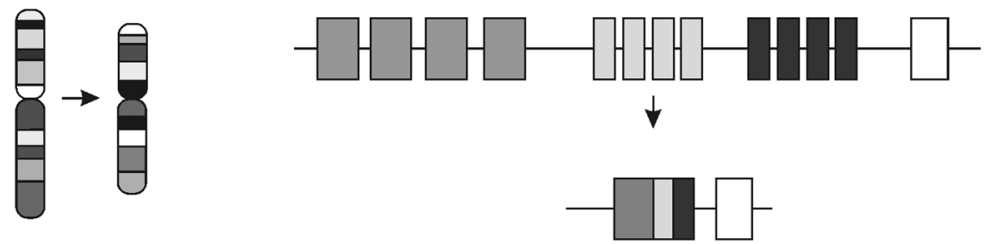

M

N
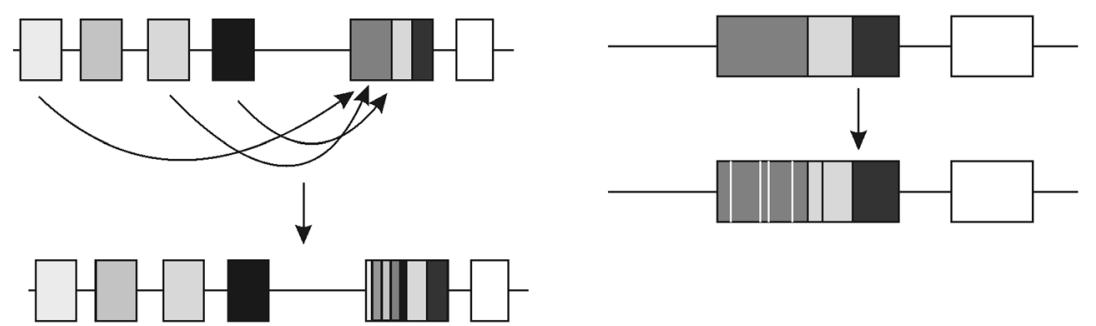

0

$\mathbf{P}$

Q

R
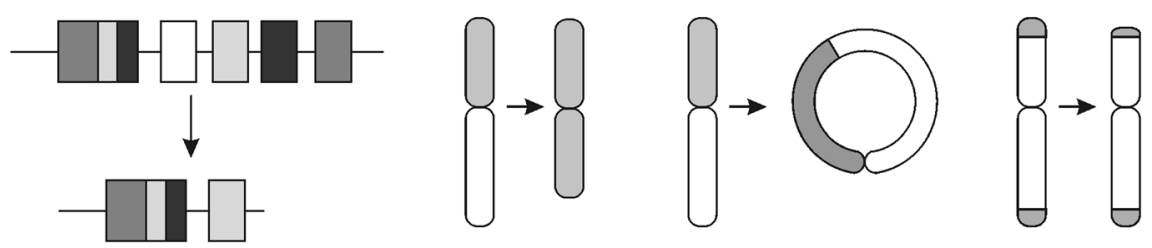

Fig. 4 Visualization of chromosomal structure variations. a Chromosome number variation, trisomy as an example. b Chromosomal amplification, more specifically copy number variation of type tandem repeat. c Intrespersed repeat chromosomal amplification. $\mathbf{d}$ Interstitial chromosomal deletion, (e) terminal deletion. There are several forms of chromosomal translocations. These include, (f) dicentric translocation, which is a form of interchromosomal translocation, (g) reciprocal translocation, h Robertsonian translocation, (i) paracentric inversion which is also intrachromosomal translocation, and (j) pericentric inversion. $\mathbf{k}$ Complex chromosomal variation. Several chromosomal changes are involved in immunological recognition molecule diversification, including (I) immunological receptor gene rearrangement, $(\mathbf{m})$ immunological receptor gene conversion, (n) somatic hypermutation, and (o) class switch recombination. The gene segments are from the left $\mathrm{V}, \mathrm{D}, \mathrm{J}$ and $\mathrm{C}$. There are up to tens of fragments in each segment type. $\mathbf{p}$ Isochromosome, (q) ring chromosome. $\mathbf{r}$ Telomere length variations, specifically telomere shortening. Note that the sizes of the telomeres in the ends of chromosomes as well as the telomere shortening are exaggerated 
and its subcategories are used to describe insertions of various mobile genetic elements. The transposition of a "DNA transposon" (VariO:0378) is catalysed by transposase enzymes with a cut-and-paste mechanism [94].

"Insertion sequence" (IS) (VariO:0392) is a short transposable element that contains only genes for transposition activity. Thereby, IS differs from other transposons that can contain or can be loaded with additional genetic material. Insertion sequence 2404 specific for Mycobacterium ulcerans originating from a crayfish can cause Buruli ulcer, a severe skin infectious disease in human [95].

"Retrotransposon insertion" (VariO:0377) means a transposon insertion via RNA intermediate which is reverse transcribed to DNA. There are three types of retrotransposons: LINE, LTR and SINE. "LINE" (VariO:0379), long interspersed element, copies constitute totally about $17 \%$ of the human genome [96]. Insertion of LINE elements of about $6000 \mathrm{bp}$ long to or close to human genes leads to a number of diseases including familiar hypocalciuric hypercalcinemia and neonatal severe hyperparathyroidism [97]. "SINE" (VariO:0380), short interspersed nuclear element, is 100-700 nucleotides long and requires LINE for replication. Alu element is the most common form of SINE and involved in numerous human diseases [98]. "LTR" (VariO:0388) (long terminal repeat) transposons form the third category of retrotransposons. They are in size between 100 and $5000 \mathrm{bp}$. Similar to SINEs, LTRs require LINE for transposition.

"Nucleotide expansion" (VariO:0430) is a large group of variations where repeated nucleotide sequences are inserted to DNA. "Microsatellite" (VariO:0188) means repetitive sequences formed by units of one to six nucleotides. CAG expansion in the HTT gene for huntingtin is an example of "trinucleotide expansion" (VariO:0189) [99]. This microsatellite expansion introduces polyglutamine tract of variable length to the amino terminus of the encoded protein. There are terms from "mononucleotide expansion" (VariO:0190) to "heptanucleotide expansion" (VariO:0452) to describe these types of variants.

"Minisatellite" (VariO:0186) is a somewhat longer repeated sequence unit, in length from 10 to $60 \mathrm{bp}$, repeated up to 50 times. $48 \mathrm{bp}$ minisatellite in dopamine receptor D4 gene, DRD4, is associated with Tourette syndrome, a neuropsychiatric disease [100].

"Type of chromosomal amplification" (VariO:0427) indicates whether the amplification is interspersed (Fig. 4c) or tandem repeat (Fig. 4b). Insertion of Alu element, a LINE transposon, is an example of "interspersed repeat" (VariO:0184), where the repeat units are separated from each other [97]. CAG trinucleotide repeat in Huntington's disease is a form of "tandem repeat" (VariO:0185) [99].
Chromosomal deletion (VariO:0193) Variants with "chromosomal deletion" (VariO:0193) are highly variable in size. "Copy number variation" (VariO:0187) can in addition to increasing copies of a DNA stretch also mean deletion. Williams-Beuren syndrome-causing deletions at $7 \mathrm{q} 11.23$ appear in the middle of the chromosome 7 [101] and are thus of "interstitial deletion" (VariO:0194) type (Fig. 4d). Deletions at chromosome 11 leading to Jacobsen syndrome are 5 to $20 \mathrm{Mb}$ long and typically include the chromosome end [102] and are thus "terminal deletion" (VariO:0195) (Fig. 4e).

Chromosomal translocation (VariO:0197) "Chromosomal translocation" (VariO:0197) rearranges genomic regions by moving them within and between chromosomes. There are several types of these changes as depicted in Fig. 4. When translocation occurs between coding regions gene fusions occur like the Philadelphia chromosome in $B C R-A B L 1$ fusion between chromosomes 9 and 22 [103], which is a hallmark of chronic myelogenous leukemia. "Interchromosomal translocation" (VariO:0202) occurs between different chromosomes, e.g. $\mathrm{t}(11 ; 14)(\mathrm{q} 13 ; \mathrm{q} 32)$ in mantle cell lymphoma patients [104]. In "dicentric translocation" (VariO:0405) both the joined segments contain a centromere (Fig. 4f). The acentric segments are lost. This kind of variation leads e.g. to Kabuki syndrome [105]. "Reciprocal chromosomal translocation" (VariO:0203) happens between two chromosomes, such as $\mathrm{t}(11 ; 14)(\mathrm{q} 13 ; \mathrm{q} 32)$ in mantle cell lymphoma patients [104] (Fig. 4g). "Robertsonian translocation" (VariO:0204) is a special type of translocation where the long arms of chromosomes are fused (Fig. 4h). This occurs between so called acrocentric chromosomes, which have very short $\mathrm{p}$ arms. In human, chromosomes 13, 14, 15, 21, 22 and $\mathrm{Y}$ are acrocentric. Infertile population has $10 \%$ increased prevalence of Robertsonian translocations compared to general population $(1 \%$ vs $0.1 \%)$. Translocation $\operatorname{rob}(14 ; 15)(\mathrm{q} 10: \mathrm{q} 10)$ is one such variation among females with recurrent abortions [106].

"Intrachromosomal translocation" (VariO:0198) occurs within one chromosome. "Chromosomal inversion" (VariO:0199) is a special type of translocation where the segment is joined inverted end to end back to the same chromosome (Fig. 4i). "Paracentric inversion" (VariO:0200) occurs within a single chromosome arm, such as in the $\mathrm{X}$-chromosome in Rett syndrome patient where the epigenetic changes lead to overexpression of $M E C P 2$ (methyl-CpG binding protein 2) gene [62] (Fig. 4i). "Pericentric inversion" (VariO:0201) includes the centromere, as an example leading to disruption of the NSD1 (nuclear receptor binding SET domain protein 1) gene in Sotos syndrome [107] (Fig. 4j). 
Complex chromosomal variation (VariO:0196) "Complex chromosomal variation" (VariO:0196) leads typically to a complex phenotype, as in the patient with myeloid leukemia associated with Down syndrome [108] (Fig. 4k).

Immunological recognition molecule diversification (VariO:0447) To achieve the huge amount of variability to immunological recognition molecules (antibodies, B and T-cell receptors, and major histocompatibility complex type I and II) special mechanisms have evolved. The human body can generate up to 10 billion different antibodies, thus effective diversity generating mechanisms are required as there are only about 22,000 genes in man.

"Immunological receptor gene rearrangement" (VariO: 0166) is the major somatic recombination step where fragments for immunological receptor genes are joined to form a gene [109] (Fig. 4l). During "immunological receptor gene conversion" (VariO:0170) secondary diversification happens by replacing homologous DNA segments [110] (Fig. 4m). During "somatic hypermutation" (VariO:0168) variations are introduced to the antigen variable region [111] (Fig. 4n).

"Class switch recombination" (VariO:0169) is the final diversification step for antibodies where immunoglobulin $\mathrm{M}$ is switched to other isotypes by changing a portion of the heavy chain coding region (see [111]) (Fig. 4o).

Isochromosome (VariO:0181) Isochromosome has one arm duplicated and the other one completely lacking (Fig. 4p). An example is the tetrasomy 18p syndrome where the isochromosome appears in addition to the normal chromosome pair [112].

Ring chromosome (VariO:0182) "Ring chromosome" has its ends joined to form a ring structure (Fig. 4q). In ring chromosome 20 syndrome patients have refractory epilepsy and other symptoms [113].

Telomere length change (VariO:0177) Telomeres are repetitive structures in the chromosome ends which are required for chromosome replication. During this process they are shortened because Okazaki fragments acting as RNA primers prevent complete replication. "Telomere extension" (VariO:0179) means variation that extends telomere [114]. In "telomere shortening" (VariO:0178) the telomere structure is shortened, a phenomenon that is related to many diseases (see [115]) (Fig. 4r).

\section{DNA sugar variation (VariO:0434)}

DNA stands for deoxyribonucleic acids. It is composed of nucleotides, deoxyribose sugars, and phosphate groups. Most DNA variations affect nucleotides, however,
"DNA sugar variation" (VariO:0434) does also exist e.g. due to carcinogens [116] and have special properties that could be beneficial for biotechnological and research applications [117].

\section{Effect on DNA tertiary structure (Vario:0171)}

DNA tertiary structure means the three-dimensional shape of the DNA. Primary structure indicates the nucleotide sequence, secondary structure the base pairing of the molecule, and quarternary structure describes intermolecular interactions or interactions with other molecules. These structural levels are analogous to protein structural levels. Experimentally determined DNA structural forms are available at ProteinData Bank (PDB) [118] and Nucleic Acid Database (NDB) [119]. The structures were visualized with Jmol: an open-source Java viewer for chemical structures in 3D (http://jmol.sourceforge.net/).

\section{Effect on DNA form (VariO:0167)}

"Effect on A-motif" (VariO:0455) is an example of "effect on DNA form" (VariO:0167), more defined as "effect on single stranded DNA structure" (VariO:0455). A-motif has a single-stranded helical structure at alkaline and neutral $\mathrm{pH}$ while at acidic $\mathrm{pH}$ it forms a right-handed helical duplex. The structure requires A-rich DNA or RNA sequence and is important e.g. for the mRNA molecules that contain long poly-A tails.

Effect on DNA double helix (VariO:0390) Most common of the DNA double helix structures is the B-form, however, there are numerous others. They have different conformations, such as A DNA [120], D DNA [121] and Z DNA [122] (see Figs. 5a to d), defined by the geometry of the DNA helices including e.g. the helix direction, rotation and number of base pairs per turn, inclination axis, rise and pitch/turn ratio etc. These molecules are right-handed except for Z-DNA which has a more open helix structure, which can be formed by alternating purine-pyrimidine sequences (Fig. 5d). These stretches can lead to the formation of deletions [123] and have an "effect on DNA double helix" (VariO:0390), more specifically "effect on Z DNA" (VariO:0421). Non B-DNA forms are involved in a number of diseases, see e.g. [124].

Effect on DNA triple helix (VariO:0175) "Effect on DNA triple helix" (VariO:0175) means alteration to triple helical nucleotide chain structure. "Effect on D loop" (VariO:0433) is a form of "effect on intermolecular DNA triple helix" (VariO:0423). In this structure the strands in double-stranded DNA are separated and one of them pairs with a third strand which can be DNA or RNA (Fig. 5e) [125]. D loops are essential for the replication of mitochondrial DNA, which is circular. Variants 
A

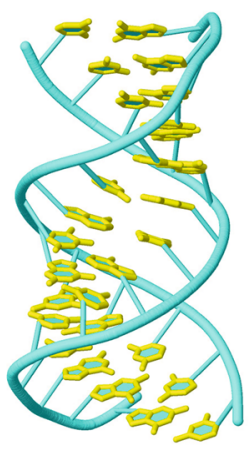

D

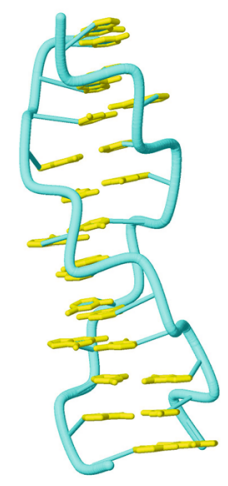

G

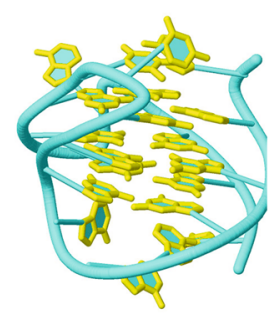

B

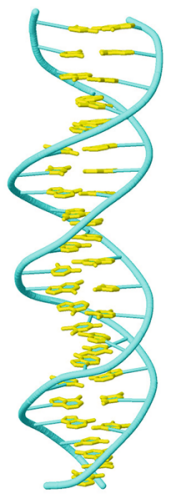

E

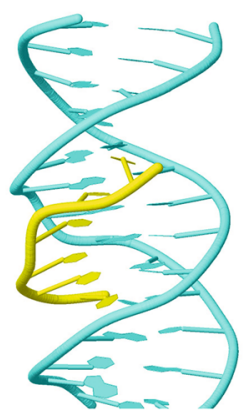

C

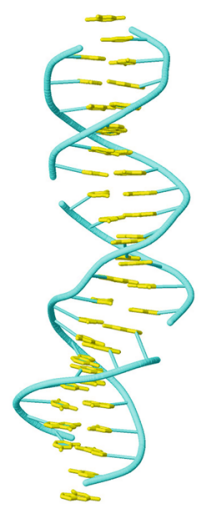

$\mathbf{F}$

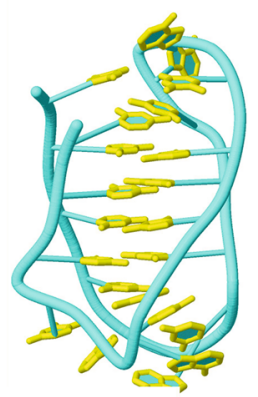

H

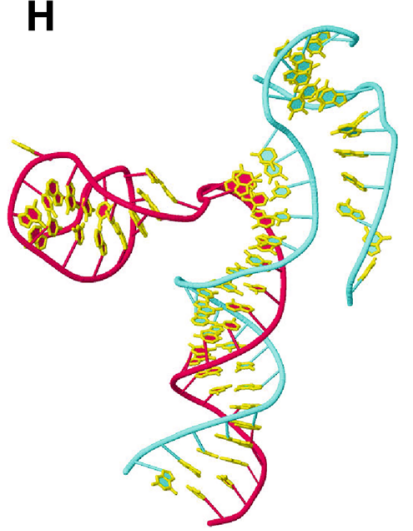

Fig. 5 Three dimensional structures of DNA forms. Double helical structures. a A DNA (5iyg) [120], b B DNA (5f9i), c D DNA (5vy6) [121], and (d) Z DNA (4ocb) [122]. e Triple helix structure (1bwg) [125]. Four-stranded DNA structures (f) i motif (PDB entry 1el2) [130], and (g) G-quadruplex (2kzd) [142]. h DNA-RNA hybrid structure of type R loop (5mga) [137]. The DNA backbone is shown in cyan and the nucleotide bases with yellow. In $\mathrm{H}$, the RNA chain backbone is in red

at the D loop are common in cancers [126] and in some other diseases.

"Effect on intramolecular DNA triple helix" (VariO: 0422) is the other type. The triple helix in $\mathrm{H}$ DNA requires mirror repeat symmetry. Supercoiling provides energy for opening of double-stranded DNA, then one of the chains swivels its background parallel to the remaining duplex DNA to form a triple helical structure. These are abundant in genomes and appear e.g. on regions that regulate expression of many genes involved in diseases. Variation can affect these structures and have "effect on H DNA" (VariO:0419) [127]. 
Effect on four-stranded DNA (VariO:0420) "Effect on four-stranded DNA" (VariO:0420) means change to DNA structures where four chains are involved. DNA cruciform is formed on inverted repeat sequences when they form a cross-shaped structure with intrastrand base pairing. There are two conformations, in extended conformation the arms are at tips of a tetrahedron, whereas in closed conformation the arms are almost parallel. Cruciforms are involved in numerous interactions at DNA usage processes including gene expression regulation, replication and recombination [128]. Variations can have "effect on DNA cruciform" (VariO:0394). Cruciform structures are prone for translocations and DNA instability [129].

i-Motifs appear in C-rich sequences. Two parallel $\mathrm{C}$-rich strands that form a duplex are intercalated in antiparallel orientation, see Fig. 5f [130]. The structures are uni-, bi-, or tetramolecular. Variations at these C-rich segments can have an "effect on i-motif" (VariO:0174). The MYC (MYC proto-oncogene, bHLH transcription factor) gene has in its promoter region seven nuclease sensitive element (NHE) $\mathrm{III}_{1}$ regions. Its expression is mainly (up to $90 \%$ ) regulated by $\mathrm{NHE} \mathrm{III}_{1}$ which can form an i-motif structure [131].

"Effect on nucleic acid G-quadruplex" (VariO:0173) describes changes where a G-quadruplex structure is involved [132] (Fig. 5g). These structures can be unimolecular, bimolecular or tetramolecular, and the chains in the two first ones can be either parallel or antiparallel, and formed by DNA, RNA or DNA-RNA hybrids [133]. Certain diseases are associated to these structures, including neurological diseases such as fragile X syndrome [134].

Effect on DNA-RNA hybrid (VariO:0424) DNA and RNA chains can bind complementarily and form hybrids. D loop is one such structure.

$\mathrm{R}$ loop consists of a DNA:RNA hybrid and a displaced single-stranded DNA. The RNA strand is produced by transcription. These loops are rather rare and instable, being targets for nuclease cleavage [135]. They are implicated in human diseases, such are trinucleotide repeat-associated diseases [136]. Changes to these hybrids can have an "effect on R loop" (VariO:0431) [137] (Fig. 5h). R-loop DB [47] includes both predicted and detected $\mathrm{R}$ loops in 8 organisms, including human.

$\mathrm{T}$ loops appear on telomeres where the single stranded chromosome terminus forms a loop to protect the DNA repair system from recognizing them [138]. T loop is part of a large complex in which several proteins are involved, in human the sheltering complex of six proteins. Variations to these structures cause "effect on T loop" (VariO:0432) [138].

\section{Epigenetic DNA modification (VariO:0156)}

Epigenetic changes are heritable traits that do not change the DNA sequence. There are three major types of "epigenetic DNA modification" (VariO:0156), including DNA methylation, histone modification and nucleosome positioning.

"Epigenetic DNA methylation" (VariO:0157) occurs almost exclusively on cytosines at $\mathrm{CpG}$ dinucleotides in $\mathrm{C}+\mathrm{G}$ rich regions called $\mathrm{CpG}$ islands. Methylations in these islands are often associated to gene silencing including genomic imprinting, which causes monoallelic gene expression. DNA methylation is significantly affected in systemic lupus erythematosus including numerous cytokine genes. An example of "epigenetic DNA methylation" (VariO:0157) is decreased methylation of the interleukin 1 receptor type 2 gene, IL1R2, which is a suppressor for IL1 signalling that leads to downregulation of IL1 and can be used as a biomarker for lupus [139]. Further, trimethylation of histone $\mathrm{H} 3$ at lysine 4 (H3K4) molecules at PTPN22 (protein tyrosine phosphatase, non-receptor type 22) and $L R P 1 B$ (LDL receptor related protein $1 \mathrm{~B}$ ) genes positively correlate with lupus severity and is annotated as "histone modification" (VariO:0453).

The GAA triplet expansion of the FXN gene in Friedreich ataxia alters nucleosome positioning and reduces transcription by making the start site not accessible [88]. This is an example of "nucleosome positioning" (VariO:0158).

\section{Genome variation (VariO:0428)}

Genome-wide alterations are described at this level.

"Chromosome set number variation" (VariO:0215) is used to annotate variations that affect the entire chromosome set number. The variations range from "nulliploidy" (VariO:0221) to polyploidy (VariO:0218), from 0 to several genomic copies, respectively. "Polyploidy" (VariO:0218) appears naturally also in some human cells including liver [68]. In "allopolyploidy" (VariO:0220) the chromosome sets originate from different organisms and is quite common in plants, such as in wheat [140]. In "autopolyploidy" (VariO:0219) the chromosome sets originate from the same organism, as in the human liver polyploidy [68].

"Complex genomic variation" (VariO:0429) describes genomic variations that contains several complex components within a single chromosome or between several ones. In chromothripsis a chromosome or several is shattered into segments some of which are randomly combined [141] and other segments are lost. This is an ultimate example of "complex genomic variation" (VariO:0429).

\section{Conclusions}

VariO facilitates a detailed description of all kinds of DNA variants and their effects and consequences. These 
annotations can be made for any organism. DNA has four major sublevels for terms: variation type, function, structure, properties. DNA molecules have four levels: DNA chain, chromosome, chromatin and genome. By combining the terms, very detailed annotations are possible. By applying Evidence \& Conclusion Ontology annotations [56] the quality and type of methods used or obtaining the data for the annotations can be described. For consistent annotation, the use of VariOtator tool [51] is recommended. It can generate variation type annotations automatically from HGVS descriptions and be directly ported to LOVD databases. Other types of annotations are made manually, VariOtator writes the annotation summary once all terms for a variant have been selected. VariO annotations will make data integration easier and more reliable. In this article, the full spectrum of DNA variations and their effects are presented in a systematic way with examples.

\section{Abbreviations}

BTK: Bruton tyrosine kinase; CNV: Copy number variation; DRD4: Dopamine receptor D4; ECO: Evidence \& Conclusion Ontology; ENCODE: The Encyclopedia of DNA Elements; FXN: Frataxin; GNA11: G protein subunit alpha 11; H3K: Histone H3 at lysine 4; HBB: Hemoglobin B; HGNC: HUGO Gene Nomenclature Committee; HGVS: Human Genome Variation Society; HTT: Huntingtin; IL1R2: Interleukin 1 receptor type 2; infA: IF1 homolog; IS: Insertion sequence; ISCN: International System for human Cytogenetic Nomenclature; LAMB1: Laminin subunit beta 1; LINE: Long interspersed element; LOVD: Leiden Open (source) Variation Database; LRP1B: LDL receptor related protein $1 B ;$ : LSDB: Locus specific variation database; LTR: Long terminal repeat; LYST: Lysosomal trafficking regulator; MECP2: Methyl-CpG binding protein 2; MYC: MYC proto-oncogene, bHLH transcription factor; NDB: Nucleic Acid Database; NHE: Nuclease sensitive element; NSD1: Nuclear receptor binding SET domain protein 1; PDB: Protein Data Bank; POLG: DNA polymerase gamma, catalytic subunit; PTPN22: Protein tyrosine phosphatase, non-receptor type 22; SINE: Short interspersed nuclear element; TAD: Topologically associating domain; TERT: Telomerase reverse transcriptase; VariO: Variation Ontology; XLA: X-linked agammagolubulinemia

\section{Acknowledgements}

Not Applicable.

\section{Funding}

This work was supported by the Swedish Research Council [VR 2015-02510] The funding body did not have any role in the design of the study and collection, analysis, or interpretation of data or in the writing of the manuscript.

\section{Availability of data and materials}

The Variation Ontology is available at http://www.variationontology.org/.

\section{Authors' contributions}

The author performed the study and wrote the manuscripts alone.The author read and approved the final manuscript.

\section{Ethics approval and consent to participate}

Not applicable.

\section{Consent for publication}

Not applicable.

\section{Competing interests}

The author declares that he has no competing interests.

\section{Publisher's Note}

Springer Nature remains neutral with regard to jurisdictional claims in published maps and institutional affiliations.

Received: 20 June 2018 Accepted: 16 November 2018

Published online: 28 December 2018

\section{References}

1. Vihinen M. Variation ontology for annotation of variation effects and mechanisms. Genome Res. 2014;24:356-64.

2. Niroula A, Vihinen M. Variation interpretation predictors: principles, types, performance, and choice. Hum Mutat. 2016;37:579-97.

3. Zhou J, Troyanskaya OG. Predicting effects of noncoding variants with deep learning-based sequence model. Nat Methods. 2015;12:931-4.

4. Consortium TEP. An integrated encyclopedia of DNA elements in the human genome. Nature. 2012;489:57-74.

5. Fu H, Zhang $X$. Noncoding variants functional prioritization methods based on predicted regulatory factor binding sites. Curr Genomics. 2017;18:322-31.

6. Ioannidis NM, Davis JR, DeGorter MK, Larson NB, McDonnell SK, French AJ, Battle AJ, Hastie TJ, Thibodeau SN, Montgomery SB, et al. FIRE: functional inference of genetic variants that regulate gene expression. Bioinformatics. 2017:33:3895-901.

7. Li S, Alvarez RV, Sharan R, Landsman D, Ovcharenko I. Quantifying deleterious effects of regulatory variants. Nucleic Acids Res. 2017;45:2307-17.

8. Folkman L, Yang Y, Li Z, Stantic B, Sattar A, Mort M, Cooper DN, Liu Y, Zhou Y. DDIG-in: detecting disease-causing genetic variations due to frameshifting indels and nonsense mutations employing sequence and structural properties at nucleotide and protein levels. Bioinformatics. 2015; 31:1599-606.

9. Zia A, Moses AM. Ranking insertion, deletion and nonsense mutations based on their effect on genetic information. BMC Bioinformatics. 2011;12:299.

10. $\mathrm{Hu} J, \mathrm{Ng}$ PC. Predicting the effects of frameshifting indels. Genome Biol. 2012;13:R9.

11. Grimm DG, Azencott CA, Aicheler F, Gieraths U, MacArthur DG, Samocha KE, Cooper DN, Stenson PD, Daly MJ, Smoller JW, et al. The evaluation of tools used to predict the impact of missense variants is hindered by two types of circularity. Hum Mutat 2015.

12. Thusberg J, Olatubosun A, Vihinen M. Performance of mutation pathogenicity prediction methods on missense variants. Hum Mutat. 2011; 32:358-68.

13. Tompitak M, Barkema GT, Schiessel H. Benchmarking and refining probability-based models for nucleosome-DNA interaction. BMC Bioinformatics. 2017;18:157.

14. Liu H, Zhang R, Xiong W, Guan J, Zhuang Z, Zhou S. A comparative evaluation on prediction methods of nucleosome positioning. Brief Bioinform. 2014;15:1014-27.

15. Dali $R, B$ Banchette $M$. A critical assessment of topologically associating domain prediction tools. Nucleic Acids Res. 2017;45:2994-3005.

16. Vihinen M. Types and effects of protein variations. Hum Genet. 2015;134:405-21.

17. Aken BL, Achuthan $P$, Akanni W, Amode MR, Bernsdorff F, Bhai J, Billis K, Carvalho-Silva D, Cummins C, Clapham P, et al. Ensembl 2017. Nucleic Acids Res. 2017:45:D635-d642.

18. Landrum MJ, Lee JM, Riley GR, Jang W, Rubinstein WS, Church DM, Maglott DR. ClinVar: public archive of relationships among sequence variation and human phenotype. Nucleic Acids Res. 2014;42:D980-5.

19. Sherry ST, Ward MH, Kholodov M, Baker J, Phan L, Smigielski EM, Sirotkin K. dbSNP: the NCBI database of genetic variation. Nucleic Acids Res. 2001;29:308-11.

20. Lek M, Karczewski KJ, Minikel EV, Samocha KE, Banks E, Fennell T, O'DonnellLuria AH, Ware JS, Hill AJ, Cummings BB, et al. Analysis of protein-coding genetic variation in 60,706 humans. Nature. 2016;536:285-91.

21. Fu W, O'Connor TD, Jun G, Kang HM, Abecasis G, Leal SM, Gabriel $S$, Rieder MJ, Altshuler D, Shendure J, et al. Analysis of 6,515 exomes reveals the recent origin of most human protein-coding variants. Nature. 2013:493:216-20.

22. Auton A, Brooks LD, Durbin RM, Garrison EP, Kang HM, Korbel JO, Marchini JL, McCarthy S, McVean GA, Abecasis GR. A global reference for human genetic variation. Nature. 2015;526:68-74.

23. Silvester N, Alako B, Amid C, Cerdeno-Tarraga A, Clarke L, Cleland I, Harrison PW, Jayathilaka S, Kay S, Keane T, et al. The European nucleotide archive in 2017. Nucleic Acids Res. 2018;46:D36-d40. 
24. Fokkema IF, Taschner PE, Schaafsma GC, Celli J, Laros JF, den Dunnen JT. LOVD v.2.0: the next generation in gene variant databases. Hum Mutat. 2011;32:557-63.

25. Beroud C, Collod-Beroud G, Boileau C, Soussi T, Junien C. UMD (universal mutation database): a generic software to build and analyze locus-specific databases. Hum Mutat. 2000;15:86-94.

26. Piirilä $H$, Väliaho J, Vihinen M. Immunodeficiency mutation databases (IDbases). Hum Mutat. 2006;27:1200-8.

27. Cheung KH, Osier MV, Kidd JR, Pakstis AJ, Miller PL, Kidd KK. ALFRED: an allele frequency database for diverse populations and DNA polymorphisms. Nucleic Acids Res. 2000;28:361-3.

28. Viennas E, Komianou A, Mizzi C, Stojiljkovic M, Mitropoulou C, Muilu J, Vihinen M, Grypioti P, Papadaki S, Pavlidis C, et al. Expanded national database collection and data coverage in the FINDbase worldwide database for clinically relevant genomic variation allele frequencies. Nucleic Acids Res. 2017;45:D846-d853.

29. Gonzalez-Galarza FF, Takeshita LY, Santos EJ, Kempson F, Maia MH, da Silva AL, Teles e Silva AL, Ghattaoraya GS, Alfirevic A, Jones AR, Middleton D. Allele frequency net 2015 update: new features for HLA epitopes, KIR and disease and HLA adverse drug reaction associations. Nucleic Acids Res. 2015:43:D784-8.

30. Dos Santos EJ, McCabe A, Gonzalez-Galarza FF, Jones AR, Middleton D. Allele frequencies net database: improvements for storage of individual genotypes and analysis of existing data. Hum Immunol. 2016;77:238-48.

31. Forbes SA, Bhamra G, Bamford S, Dawson E, Kok C, Clements J, Menzies A, Teague JW, Futreal PA, Stratton MR. The Catalogue of Somatic Mutations in Cancer (COSMIC). Curr Protoc Hum Genet. 2008;Chapter 10:Unit 10.11.

32. Tomczak K, Czerwinska P, Wiznerowicz M. The Cancer genome atlas (TCGA): an immeasurable source of knowledge. Contemp Oncol (Pozn). 2015;19: A68-77.

33. Zhang J, Baran J, Cros A, Guberman JM, Haider S, Hsu J, Liang Y, Rivkin E, Wang J, Whitty B, et al. International Cancer Genome Consortium Data Portal--a one-stop shop for cancer genomics data. Database (Oxford) 2011; 2011. p. bar026

34. Qasim I, Ahmad B, Khan MA, Khan N, Muhammad N, Basit S, Khan S. Pakistan genetic mutation database (PGMD); a centralized Pakistani mutome data source. Eur J Med Genet. 2017:61:204-8.

35. Tan EC, Loh M, Chuon D, Lim YP. Singapore human mutation/ polymorphism database: a country-specific database for mutations and polymorphisms in inherited disorders and candidate gene association studies. Hum Mutat. 2006;27:232-5.

36. Phan L, Hsu J, Tri LQ, Willi M, Mansour T, Kai Y, Garner J, Lopez J, Busby B. dbVar structural variant cluster set for data analysis and variant comparison. F1000Res. 2016:5:673.

37. MacDonald JR, Ziman R, Yuen RK, Feuk L, Scherer SW. The database of genomic variants: a curated collection of structural variation in the human genome. Nucleic Acids Res. 2014;42:D986-92.

38. Lappalainen I, Lopez J, Skipper L, Hefferon T, Spalding JD, Garner J, Chen C, Maguire M, Corbett M, Zhou G, et al. DbVar and DGVa: public archives for genomic structural variation. Nucleic Acids Res. 2013:41:D936-41.

39. Martinez-Fundichely A, Casillas S, Egea R, Ramia M, Barbadilla A, Pantano L, Puig M, Caceres M. InvFEST, a database integrating information of polymorphic inversions in the human genome. Nucleic Acids Res. 2014;42 D1027-32.

40. Mir AA, Philippe C, Cristofari G. euL1db: the European database of L1HS retrotransposon insertions in humans. Nucleic Acids Res. 2015;43:D43-7.

41. Penzkofer $T$, Jager M, Figlerowicz M, Badge R, Mundlos S, Robinson PN, Zemojtel T. L1Base 2: more retrotransposition-active LINE-1s, more mammalian genomes. Nucleic Acids Res. 2017:45:D68-d73.

42. Vassetzky NS, Kramerov DA. SINEBase: a database and tool for SINE analysis. Nucleic Acids Res. 2013:41:D83-9.

43. Ruitberg CM, Reeder DJ, Butler JM. STRBase: a short tandem repeat DNA database for the human identity testing community. Nucleic Acids Res. 2001;29:320-2

44. Lebron R, Gomez-Martin C, Carpena P, Bernaola-Galvan P, Barturen G, Hackenberg M, Oliver JL. NGSmethDB 2017: Enhanced methylomes and differential methylation. Nucleic Acids Res. 2017:(45):D97-d103.

45. Ziebarth JD, Bhattacharya A, Chen A, Cui Y. PolymiRTS database 2.0: linking polymorphisms in microRNA target sites with human diseases and complex traits. Nucleic Acids Res. 2012;40:D216-21.
46. Bhattacharya A, Cui Y. SomamiR 2.0: a database of cancer somatic mutations altering microRNA-ceRNA interactions. Nucleic Acids Res. 2016:44: D1005-10.

47. Jenjaroenpun $P$, Wongsurawat $T$, Sutheeworapong S, Kuznetsov VA. RloopDB: a database for R-loop forming sequences (RLFS) and R-loops. Nucleic Acids Res. 2017:45:D119-d127.

48. Leroy B, Anderson M, Soussi T. TP53 mutations in human cancer: database reassessment and prospects for the next decade. Hum Mutat. 2014;35:672-88.

49. Members BDC. Database resources of the BIG data center in 2018. Nucleic Acids Res. 2018;46:D14-d20.

50. Vihinen M. Variation ontology: annotator guide. J Biomed Semantics. 2014;5:9.

51. Schaafsma GC, Vihinen M. VariOtator, a software tool for variation annotation with the variation ontology. Hum Mutat. 2016;37:344-9.

52. Väliaho J, Smith CIE, Vihinen M. BTKbase: the mutation database for X-linked agammaglobulinemia. Hum Mutat. 2006;27:1209-17.

53. Lappalainen I, Thusberg J, Shen B, Vihinen M. Genome wide analysis of pathogenic SH2 domain mutations. Proteins. 2008;72:779-92.

54. Famiglietti ML, Estreicher A, Gos A, Bolleman J, Gehant S, Breuza L, Bridge A Poux S, Redaschi N, Bougueleret L, Xenarios I. Genetic variations and diseases in UniProtKB/Swiss-Prot: the ins and outs of expert manual curation. Hum Mutat. 2014;35:927-35.

55. Nair PS, Vihinen M. VariBench: a benchmark database for variations. Hum Mutat. 2013;34:42-9.

56. Chibucos MC, Mungall CJ, Balakrishnan R, Christie KR, Huntley RP, White O, Blake JA, Lewis SE, Giglio M. Standardized description of scientific evidence using the evidence ontology (ECO). Database (Oxford). 2014;2014:bau066.

57. den Dunnen JT, Antonarakis SE. Nomenclature for the description of human sequence variations. Hum Genet. 2001;109:121-4.

58. McGowan-Jordan J, Schmid M, Simons A: ISCN 2016: an international system for human Cytogenomic nomenclature. S. Karger AG; 2016.

59. Gray KA, Yates B, Seal RL, Wright MW, Bruford EA. Genenames.org: the HGNC resources in 2015. Nucleic Acids Res. 2015:43:D1079-85.

60. Ferraro A. Altered primary chromatin structures and their implications in cancer development. Cell Oncol (Dordr). 2016;39:195-210.

61. Megarbane A, Ravel A, Mircher C, Sturtz F, Grattau Y, Rethore MO, Delabar JM, Mobley WC. The 50th anniversary of the discovery of trisomy 21: the past, present, and future of research and treatment of Down syndrome. Genet Med. 2009;11:611-6.

62. Vieira JP, Lopes F, Silva-Fernandes A, Sousa MV, Moura S, Sousa S, Costa BM, Barbosa M, Ylstra B, Temudo T, et al. Variant Rett syndrome in a girl with a pericentric X-chromosome inversion leading to epigenetic changes and overexpression of the MECP2 gene. Int J Dev Neurosci. 2015:46:82-7.

63. Holinski-Feder E, Weiss M, Brandau O, Jedele KB, Nore B, Bäckesjö CM, Vihinen M, Hubbard SR, Belohradsky BH, Smith Cl, Meindl A. Mutation screening of the BTK gene in 56 families with X-linked agammaglobulinemia (XLA): 47 unique mutations without correlation to clinical course. Pediatrics. 1998;101:276-84

64. Vorechovsky I, Luo L, Hertz JM, Froland SS, Klemola T, Fiorini M, Quinti I, Paganelli R, Ozsahin H, Hammarstrom L, et al. Mutation pattern in the Bruton's tyrosine kinase gene in 26 unrelated patients with X-linked agammaglobulinemia. Hum Mutat. 1997;9:418-25.

65. Jin H, Webster AD, Vihinen M, Sideras P, Vorechovsky I, Hammarstrom L, Bernatowska-Matuszkiewicz E, Smith Cl, Bobrow M, Vetrie D. Identification of Btk mutations in 20 unrelated patients with X-linked agammaglobulinaemia (XLA). Hum Mol Genet. 1995:4:693-700.

66. Vihinen M, Nore BF, Mattsson PT, Bäckesjö CM, Nars M, Koutaniemi S, Watanabe C, Lester T, Jones A, Ochs HD, Smith Cl. Missense mutations affecting a conserved cysteine pair in the TH domain of Btk. FEBS Lett. 1997; 413:205-10

67. Alsmadi O, John SE, Thareja G, Hebbar P, Antony D, Behbehani K, Thanaraj TA. Genome at juncture of early human migration: a systematic analysis of two whole genomes and thirteen exomes from Kuwaiti population subgroup of inferred Saudi Arabian tribe ancestry. PLoS One. 2014:9:e99069.

68. Gentric G, Desdouets C. Polyploidization in liver tissue. Am J Pathol. 2014; 184:322-31.

69. Okoh MP, Kainulainen L, Heiskanen K, Isa MN, Varming K, Ruuskanen O, Vihinen M. Novel insertions of Bruton tyrosine kinase in patients with Xlinked agammaglobulinemia. Hum Mutat. 2002;20:480-1. 
70. Curtis SK, Hebert MD, Saha BK. Twin carriers of X-linked agammaglobulinemia (XLA) due to germline mutation in the Btk gene. Am J Med Genet. 2000;90:229-32.

71. Landis CA, Masters SB, Spada A, Pace AM, Bourne HR, Vallar L. GTPase inhibiting mutations activate the alpha chain of Gs and stimulate adenylyl cyclase in human pituitary tumours. Nature. 1989;340:692-6.

72. Mattsson PT, Lappalainen I, Bäckesjö CM, Brockmann E, Lauren S, Vihinen M, Smith CIE. Six X-linked agammaglobulinemia-causing missense mutations in the Src homology 2 domain of Bruton's tyrosine kinase: phosphotyrosinebinding and circular dichroism analysis. J Immunol. 2000;164:4170-7.

73. Liang $P$, Ding $C$, Sun $H$, Xie X, Xu Y, Zhang X, Sun Y, Xiong Y, Ma W, Liu Y, et al. Correction of beta-thalassemia mutant by base editor in human embryos. Protein Cell. 2017;8:811-22.

74. Javierre BM, Fernandez AF, Richter J, Al-Shahrour F, Martin-Subero Jl, Rodriguez-Ubreva J, Berdasco M, Fraga MF, O'Hanlon TP, Rider LG, et al. Changes in the pattern of DNA methylation associate with twin discordance in systemic lupus erythematosus. Genome Res. 2010;20:170-9.

75. Jaloszynski P, Masutani C, Hanaoka F, Perez AB, Nishimura S. 8-Hydroxyguanine in a mutational hotspot of the c-ha-ras gene causes misreplication, 'action-at-adistance' mutagenesis and inhibition of replication. Nucleic Acids Res. 2003;31: 6085-95.

76. Fresco JR, Amosova O. Site-Specific Self-Catalyzed DNA Depurination: a biological mechanism that leads to mutations and creates sequence diversity. Annu Rev Biochem. 2017;86:461-84.

77. Chen J, Furano AV. Breaking bad: the mutagenic effect of DNA repair. DNA Repair (Amst). 2015;32:43-51.

78. Poncz M, Ballantine M, Solowiejczyk D, Barak I, Schwartz E, Surrey S. Betathalassemia in a Kurdish Jew. Single base changes in the T-A-T-A box. J Biol Chem. 1982;257:5994-6.

79. Ganai RA, Johansson E. DNA replication-a matter of Fidelity. Mol Cell. 2016; 62:745-55.

80. Liu B, Xue Q, Tang Y, Cao J, Guengerich FP, Zhang H. Mechanisms of mutagenesis: DNA replication in the presence of DNA damage. Mutat Res Rev Mutat Res. 2016;768:53-67.

81. Horn S, Figl A, Rachakonda PS, Fischer C, Sucker A, Gast A, Kadel S, Moll I, Nagore $E$, Hemminki $K$, et al. TERT promoter mutations in familial and sporadic melanoma. Science. 2013;339:959-61.

82. Conley ME, Mathias D, Treadaway J, Minegishi Y, Rohrer J. Mutations in btk in patients with presumed X-linked agammaglobulinemia. Am J Hum Genet. 1998;62:1034-43.

83. Shen B, Vihinen M. Conservation and covariance in PH domain sequences: physicochemical profile and information theoretical analysis of XLA-causing mutations in the Btk PH domain. Protein Eng Des Sel. 2004;17:267-76.

84. Van Goethem G, Dermaut B, Lofgren A, Martin JJ, Van Broeckhoven C. Mutation of POLG is associated with progressive external ophthalmoplegia characterized by mtDNA deletions. Nat Genet. 2001;28:211-2.

85. Landau AM, Pacheco MG, Prina AR. A second infA plastid gene point mutation shows a compensatory effect on the expression of the cytoplasmic line 2 (CL2) syndrome in barley. J Hered. 2011;102:633-9.

86. Taylor DE, Levine JG. Characterization of a plasmid mutation affecting maintenance, transfer and elimination by novobiocin. Mol Gen Genet. 1979; 174:127-33

87. Cohen S, Segal D. Extrachromosomal circular DNA in eukaryotes: possible involvement in the plasticity of tandem repeats. Cytogenet Genome Res. 2009:124:327-38.

88. Chutake YK, Costello WN, Lam C, Bidichandani SI. Altered nucleosome positioning at the transcription start site and deficient transcriptional initiation in Friedreich ataxia. J Biol Chem. 2014;289:15194-202.

89. Kaiser VB, Semple CA. When TADs go bad: chromatin structure and nuclear organisation in human disease. F1000Res. 2017;6:314

90. Angulo MA, Butler MG, Cataletto ME. Prader-Willi syndrome: a review of clinical, genetic, and endocrine findings. J Endocrinol Investig. 2015;38: 1249-63.

91. Manoli I, Golas G, Westbroek W, Vilboux T, Markello TC, Introne W, Maynard D, Pederson B, Tsilou E, Jordan MB, et al. Chediak-Higashi syndrome with early developmental delay resulting from paternal heterodisomy of chromosome 1. Am J Med Genet A. 2010;152a:1474-83.

92. Darcy D, Atwal PS, Angell C, Gadi I, Wallerstein R. Mosaic paternal genomewide uniparental isodisomy with down syndrome. Am J Med Genet A. 2015;167a:2463-9
93. Padiath QS, Saigoh K, Schiffmann R, Asahara H, Yamada T, Koeppen A Hogan K, Ptacek LJ, Fu YH. Lamin B1 duplications cause autosomal dominant leukodystrophy. Nat Genet. 2006;38:1114-23.

94. Munoz-Lopez M, Garcia-Perez JL. DNA transposons: nature and applications in genomics. Curr Genomics. 2010;11:115-28.

95. Ohtsuka M, Kikuchi N, Yamamoto T, Suzutani T, Nakanaga K, Suzuki K, Ishii $\mathrm{N}$. Buruli ulcer caused by Mycobacterium ulcerans subsp shinshuense: a rare case of familial concurrent occurrence and detection of insertion sequence 2404 in Japan. JAMA Dermatol. 2014;150:64-7.

96. Doucet AJ, Hulme AE, Sahinovic E, Kulpa DA, Moldovan JB, Kopera HC, Athanikar JN, Hasnaoui M, Bucheton A, Moran JV, Gilbert N. Characterization of LINE-1 ribonucleoprotein particles. PLoS Genet. 2016:6(10):e1001150.

97. Janicic N, Pausova Z, Cole DE, Hendy GN. Insertion of an Alu sequence in the $\mathrm{ca}(2+)$-sensing receptor gene in familial hypocalciuric hypercalcemia and neonatal severe hyperparathyroidism. Am J Hum Genet. 1995:56:880-6.

98. Hancks DC, Kazazian HH Jr. Roles for retrotransposon insertions in human disease. Mob DNA. 2016:7:9.

99. Walker FO. Huntington's disease. Lancet. 2007;369:218-28.

100. Liu S, Cui J, Zhang X, Wu W, Niu H, Ma X, Xu H, Yi M. Variable number tandem repeats in dopamine receptor D4 in Tourette's syndrome. Mov Disord. 2014;29:1687-91.

101. Bayes M, Magano LF, Rivera N, Flores R, Perez Jurado LA. Mutational mechanisms of Williams-Beuren syndrome deletions. Am J Hum Genet. 2003;73:131-51.

102. Dalm VA, Driessen GJ, Barendregt BH, van Hagen PM, van der Burg M. The $11 \mathrm{q}$ terminal deletion disorder Jacobsen syndrome is a syndromic primary Immunodeficiency. J Clin Immunol. 2015;35:761-8.

103. Nowell PC, Hungerford DA. Chromosome studies on normal and leukemic human leukocytes. J Natl Cancer Inst. 1960;25:85-109.

104. Li JY, Gaillard F, Moreau A, Harousseau JL, Laboisse C, Milpied N, Bataille R, Avet-Loiseau $H$. Detection of translocation $t(11 ; 14)(q 13 ; q 32)$ in mantle cell lymphoma by fluorescence in situ hybridization. Am J Pathol. 1999;154: 1449-52.

105. Lynch SA, Ashcroft KA, Zwolinski S, Clarke C, Burn J. Kabuki syndrome-like features in monozygotic twin boys with a pseudodicentric chromosome 13. J Med Genet. 1995:32:227-30.

106. Ananthapur V, Avvari S, Tella S, Nallari P, Akka J. A Robertsonian translocation rob $(14 ; 15)$ (q10:q10) in a patient with recurrent abortions: a case report. J Reprod Infertil. 2010;11:197-200.

107. Malan V, De Blois MC, Prieur M, Perrier-Waill MC, Huguet-Nedjar C, Gegas L, Turleau C, Vekemans M, Munnich A, Romana SP. Sotos syndrome caused by a paracentric inversion disrupting the NSD1 gene. Clin Genet. 2008;73:89-91.

108. de Souza DC, de Figueiredo AF, Ney Garcia DR, da Costa ES, Othman MAK, Liehr T, Abdelhay E, Silva MLM, de Souza Fernandez T. A unique set of complex chromosomal abnormalities in an infant with myeloid leukemia associated with Down syndrome. Mol Cytogenet. 2017;10:35.

109. Roth DB. V(D)J recombination: mechanism, errors, and Fidelity. Microbiol Spectr. 2014:2(6):MNDA3-0041-2014.

110. Darlow JM, Stott DI. Gene conversion in human rearranged immunoglobulin genes. Immunogenetics. 2006;58:511-22.

111. Methot SP, Di Noia JM. Molecular mechanisms of somatic Hypermutation and class switch recombination. Adv Immunol. 2017;133:37-87.

112. Plaiasu V, Ochiana D, Motei G, Georgescu A. A rare chromosomal disorder isochromosome 18p syndrome. Maedica (Buchar). 2011;6:132-6.

113. Daber RD, Conlin LK, Leonard LD, Canevini MP, Vignoli A, Hosain S, Brown LW, Spinner NB. Ring chromosome 20. Eur J Med Genet. 2012;55:381-7.

114. Hannes F, Van Houdt J, Quarrell OW, Poot M, Hochstenbach R, Fryns JP, Vermeesch JR. Telomere healing following DNA polymerase arrest-induced breakages is likely the main mechanism generating chromosome $4 p$ terminal deletions. Hum Mutat. 2010;31:1343-51.

115. Kong CM, Lee XW, Wang X. Telomere shortening in human diseases. FEBS J. 2013:280:3180-93.

116. Hiramoto K, Kaku M, Sueyoshi A, Fujise M, Kikugawa K. DNA base and deoxyribose modification by the carbon-centered radical generated from 4(hydroxymethyl)benzenediazonium salt, a carcinogen in mushroom. Chem Res Toxicol. 1995:8:356-62.

117. Suresh G, Priyakumar UD. Atomistic investigation of the effect of incremental modification of deoxyribose sugars by locked nucleic acid (beta-D-LNA and alpha-L-LNA) moieties on the structures and thermodynamics of DNA-RNA hybrid duplexes. J Phys Chem B. 2014;118: 5853-63. 
118. Berman HM, Westbrook J, Feng Z, Gilliland G, Bhat TN, Weissig H, Shindyalov IN, Bourne PE. The Protein Data Bank. Nucleic Acids Res. 2000;28:235-42.

119. Berman HM, Olson WK, Beveridge DL, Westbrook J, Gelbin A, Demeny T, Hsieh SH, Srinivasan AR, Schneider B. The nucleic acid database. A comprehensive relational database of three-dimensional structures of nucleic acids. Biophys J. 1992;63:751-9.

120. Karthik S, Thirugnanasambandam A, Mandal PK, Gautham N. Comparison of X-ray crystal structures of a tetradecamer sequence d(CCCGGGTACCCGGG)2 at 1.7 a resolution. Nucleosides Nucleotides Nucleic Acids. 2017:36:343-54.

121. Simmons CR, Zhang F, MacCulloch T, Fahmi N, Stephanopoulos N, Liu Y, Seeman NC, Yan $\mathrm{H}$. Tuning the cavity size and chirality of self-assembling 3D DNA crystals. J Am Chem Soc. 2017;139:11254-60.

122. Luo Z, Dauter M, Dauter Z. Phosphates in the Z-DNA dodecamer are flexible, but their P-SAD signal is sufficient for structure solution. Acta Crystallogr D Biol Crystallogr. 2014;70:1790-800.

123. Wang G, Christensen LA, Vasquez KM. Z-DNA-forming sequences generate large-scale deletions in mammalian cells. Proc Natl Acad Sci U S A. 2006; 103:2677-82.

124. Bacolla A, Wells RD. Non-B DNA conformations as determinants of mutagenesis and human disease. Mol Carcinog. 2009;48:273-85.

125. Asensio $\mathrm{JL}$, Brown T, Lane AN. Solution conformation of a parallel DNA triple helix with $5^{\prime}$ and $3^{\prime}$ triplex-duplex junctions. Structure. 1999;7:1-11.

126. Sharma H, Singh A, Sharma C, Jain SK, Singh N. Mutations in the mitochondrial DNA D-loop region are frequent in cervical cancer. Cancer Cell Int. 2005;5:34.

127. Wang G, Vasquez KM. Naturally occurring H-DNA-forming sequences are mutagenic in mammalian cells. Proc Natl Acad Sci U S A. 2004;101:13448-53.

128. Brazda V, Laister RC, Jagelska EB, Arrowsmith C. Cruciform structures are a common DNA feature important for regulating biological processes. BMC Mol Biol. 2011;12:33.

129. Inagaki H, Ohye T, Kogo H, Kato T, Bolor H, Taniguchi M, Shaikh TH, Emanuel BS, Kurahashi H. Chromosomal instability mediated by non-B DNA: cruciform conformation and not DNA sequence is responsible for recurrent translocation in humans. Genome Res. 2009;19:191-8.

130. Phan AT, Gueron M, Leroy JL. The solution structure and internal motions of a fragment of the cytidine-rich strand of the human telomere. J Mol Biol. 2000;299:123-44.

131. Dai J, Hatzakis E, Hurley LH, Yang D. I-motif structures formed in the human C-MYC promoter are highly dynamic--insights into sequence redundancy and I-motif stability. PLoS One. 2010;5:e11647.

132. Biffi G, Tannahill D, McCafferty J, Balasubramanian S. Quantitative visualization of DNA G-quadruplex structures in human cells. Nat Chem. 2013:5:182-6.

133. Simone R, Fratta P, Neidle S, Parkinson GN, Isaacs AM. G-quadruplexes: emerging roles in neurodegenerative diseases and the non-coding transcriptome. FEBS Lett. 2015;589:1653-68.

134. Maizels N. G4-associated human diseases. EMBO Rep. 2015;16:910-22.

135. Freudenreich $\mathrm{CH}$. R-loops: targets for nuclease cleavage and repeat instability. Curr Genet. 2018;64:789-94.

136. Richard P, Manley JL. R loops and links to human disease. J Mol Biol. 2017: 429:3168-80.

137. Stella S, Alcon P, Montoya G. Structure of the Cpf1 endonuclease R-loop complex after target DNA cleavage. Nature. 2017;546:559-63.

138. de Lange T. T-loops and the origin of telomeres. Nat Rev Mol Cell Biol. 2004;5:323-9.

139. Wang Z, Chang C, Peng M, Lu Q. Translating epigenetics into clinic: focus on lupus. Clin Epigenetics. 2017;9:78.

140. Matsuoka Y, Takumi S, Nasuda S. Genetic mechanisms of allopolyploid speciation through hybrid genome doubling: novel insights from wheat (Triticum and Aegilops) studies. Int Rev Cell Mol Biol. 2014;309:199-258.

141. Ly P, Cleveland DW. Rebuilding chromosomes after catastrophe: emerging mechanisms of Chromothripsis. Trends Cell Biol. 2017;27:917-30.

142. Lim KW, Lacroix L, Yue DJ, Lim JK, Lim JM, Phan AT. Coexistence of two distinct G-quadruplex conformations in the hTERT promoter. J Am Chem Soc. 2010;132:12331-42.

Ready to submit your research? Choose BMC and benefit from:

- fast, convenient online submission

- thorough peer review by experienced researchers in your field

- rapid publication on acceptance

- support for research data, including large and complex data types

- gold Open Access which fosters wider collaboration and increased citations

- maximum visibility for your research: over $100 \mathrm{M}$ website views per year

At BMC, research is always in progress.

Learn more biomedcentral.com/submissions 